\title{
Is Assessment of Absolute Myocardial Perfusion with SPECT Ready for Prime Time?
}

Q uantification of absolute myocardial flow and flow reserve using dynamic PET imaging has now been more widely applied clinically to detect microvascular disease, improve diagnostic accuracy for detection of coronary artery disease, and improve prognostic implications of stress perfusion imaging in patients with heart disease (1). PET imaging offers some distinct advantages over SPECT imaging for the assessment of absolute myocardial flow, including higher count sensitivity and higher spatial resolution than SPECT; well-established approaches for attenuation correction for all PET imaging systems; and approved radiotracers with favorable kinetics for the assessment of absolute myocardial perfusion. The clinical quantification of myocardial flow with PET has been facilitated by the availability of several Food and Drug Administration-approved quantitative software programs that are commercially available. These programs have been shown to be highly reproducible and comparable for estimation of myocardial flow reserve in patients, although some differences exist among these programs for estimation of absolute myocardial flow (2). Although the availability of hybrid PET/CT scanners is growing,

\section{See page 1685}

there remains limited access to existing PET systems for cardiac imaging along with reimbursement hurdles.

Currently, most nuclear cardiac imaging is performed on SPECT imaging systems, which outnumber the PET systems by at least 10 to 1 , so the potential to perform dynamic SPECT cardiac imaging for determination of absolute myocardial flow holds tremendous clinical benefit. Many studies have proposed the use of serial SPECT imaging for the determination of myocardial flow reserve, using conventional cameras with $\mathrm{NaI}$ detectors and approved clinical SPECT perfusion agents $(3,4)$ and more recently with high-sensitivity dedicated cardiac SPECT systems with solid-state cadmiumzinc-telluride (CZT) detectors capable of list-mode data acquisitions (5). In the current issue of the Journal of Nuclear Medicine, Wells et al. (6) use a unique stationary solid-state CZT camera with the capacity for dynamic SPECT imaging for the determination of absolute flow using both ${ }^{201} \mathrm{Tl}$ and ${ }^{99 \mathrm{~m}} \mathrm{Tc}$-labeled radiotracers in pigs both at rest and during stress with transient coronary

Received Aug. 23, 2014; revision accepted Aug. 25, 2014.

For correspondence or reprints contact: Albert J. Sinusas, Yale University School of Medicine, 789 Howard Ave., DANA 3, P.O. Box 208017, New Haven, CT 06510-8017.

E-mail: albert.sinusas@yale.edu

Published online Sep. 18, 2014.

COPYRIGHT (c) 2014 by the Society of Nuclear Medicine and Molecular Imaging, Inc.

DOI: $10.2967 /$ jnumed.114.144550 occlusion. This pilot preclinical study demonstrates the feasibility of dynamic SPECT imaging for quantification of absolute myocardial flow.

Earlier studies using conventional SPECT cameras assumed that the radiotracers are retained in the myocardium and therefore estimated myocardial flow by computing the ratio of the counts in the tissue over the integral of the arterial concentration of the tracer up to the time of SPECT imaging $(4,7)$. Some investigators applied planar imaging to define the arterial input function and delayed SPECT imaging to define the myocardial uptake, whereas others used multidetector SPECT cameras to obtain short (ranging from $10 \mathrm{~s}$ to $2 \mathrm{~min}$ ) serial SPECT acquisitions with continuous back and forth camera rotations over a $180^{\circ}$ arch. The dynamic cardiac SPECT approaches estimated washin and washout kinetics from a series of reconstructed SPECT images, applying compartmental analysis similar to dynamic PET imaging. The earlier dynamic SPECT studies were performed with ${ }^{201} \mathrm{Tl}$ (8), whereas later studies used ${ }^{99 \mathrm{~m}} \mathrm{Tc}$-labeled perfusion agents having a high first-pass extraction, for example, ${ }^{99 \mathrm{~m}}$ Tc-teboroxime (9). Iida et al., who pioneered this work, evaluated many potential serial SPECT acquisition schemes (8). Unfortunately, when rotating gamma cameras are used to image an emission source, the activity will vary significantly during the time it takes for the camera to complete a rotation, resulting in inconsistent angular projections (3). These inconsistent projections can lead to biases in the estimation of kinetic parameters. A better method to analyze dynamic SPECT data acquired this way involves estimation of the kinetic parameters directly from the dynamic projection data $(3,10)$. These approaches using rotating cameras when applied to both rest and stress injections of radiotracer were best used for estimation of myocardial flow reserve as opposed to calculation of true absolute myocardial flow.

Ben Haim et al. used an upright solid-state CZT SPECT camera with a configuration and operation of the detectors slightly different from that used by Wells et al. to perform dynamic SPECT imaging in 95 patients to derive a retention index for estimation of regional and global myocardial perfusion reserve (5). To avoid the problem of truncated projections, this camera sweeps the direction of the individual CZT detector modules over the subject. However, this dynamic sweeping motion of the detectors introduces temporal variation in the projections, also complicating the reconstruction. These investigators also did not apply attenuation or scatter correction and therefore could not derive a true estimate of absolute myocardial flow.

The study by Wells et al. evaluated each of the currently used

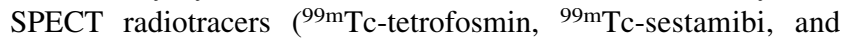
${ }^{201} \mathrm{Tl}$ ), although the authors performed a limited number of studies with each of these agents under a limited number of experimental conditions. The use of a model of transient left anterior descending artery occlusion in combination with pharmacologic stress while creating a range of flows within the myocardium actually complicates their analyses. The blood clearance for 
${ }^{201} \mathrm{Tl}$ and the ${ }^{99 \mathrm{~m}} \mathrm{Tc}-$ labeled radiotracers is relatively slow, and significant redistribution of radiotracer within the ischemic area after reperfusion might occur, because the short period of coronary occlusion followed by reperfusion would result in reactive hyperemia even in the presence of dipyridamole stress. This change in flow during the time of radiotracer extraction could have influenced their results, because extraction of these radiotracers is dependent on flow. Extraction and clearance of these tracers are also dependent on cellular viability, although the effect of myocardial viability on estimation of flow was not evaluated in the current study. The authors suggest that a 1-tissue compartmental model may be sufficient in evaluation of these radiotracers. A 1-tissue-compartment model may not hold true for ${ }^{201} \mathrm{Tl}$, because the clearance kinetics of ${ }^{201} \mathrm{Tl}$ are typically biexponential, with the initial uptake and clearance involving the exchange between intravascular, interstitial, and cellular compartments. The relative importance and value of a 2-tissue compartmental model may prove to be more important under more complex clinical conditions in which normal tissue may be admixed with ischemic or injured tissue. Therefore, the relative value of their 1- or 2-tissue compartmental models for future clinical application cannot be established on the basis of the limited experimental conditions tested in the current study. Despite these limitations, ${ }^{201} \mathrm{Tl}$ does seem to provide a more accurate estimate of absolute myocardial flow over the existing ${ }^{99 \mathrm{~m}}$ Tc-labeled perfusion agents.

To accurately estimate parameters through kinetic modeling of a 4-dimensional dynamic SPECT dataset, the prerequisite is to obtain an absolute quantitative reconstruction of each 3-dimensional SPECT image first. Currently, there are several technical issues that need to be addressed to further improve the quantitative accuracy of dedicated cardiac SPECT systems for the determination of absolute myocardial flow.

The first technical issue is scatter correction. Pixelated CZT detectors provide energy resolution superior to that of conventional $\mathrm{NaI}$ detectors. However, because of incomplete charge collection and interdetector scatter, a low-energy tail is present on the energy spectrum of CZT detectors. This low-energy tail, known as the spillover effect, will complicate energy windowbased scatter correction. It has been shown that applying the conventional triple-energy-window method to images obtained on an upright cardiac CZT SPECT system (D-SPECT; Spectrum Dynamic Medical) resulted in overcorrection (11). Therefore, the unique spillover effect needs to be characterized first and incorporated into scatter correction for CZT SPECT. For the cardiac CZT SPECT scanner (Discovery NM 530c; GE Healthcare) with pinhole collimators used in the study by Wells et al., the spillover correction model needs to be more sophisticated than the previously published method for the CZT SPECT scanner with parallelhole collimators, because the pinhole collimator geometry introduces additional incident angle-dependent spillover effect. Our group recently introduced an approach to improve the scatter correction for CZT SPECT images acquired with pinhole collimators (12), although further optimization and validation studies of this approach are needed.

The second technical issue is projection truncation. For the truly stationary CZT pinhole SPECT system used in the study by Wells et al., all the pinhole collimators focus on a field of view of approximately $19 \mathrm{~cm}$, a configuration that facilitates focused dynamic imaging of the heart. However, the radioactivity in adjacent organs and background may be truncated, and this truncation could cause substantial quantitative errors. Previous studies have shown that using a small reconstruction matrix could lead to overestimation of quantification, whereas increasing the reconstruction matrix size can minimize this overestimation (13). Although the previous study demonstrated that incorporating body contour obtained from CT as a prior information in SPECT reconstruction can provide the most accurate quantification, this will require either $\mathrm{CT}$ images obtained from a hybrid SPECT/CT system or registered CT images acquired on another scanner, as used in the study by Wells et al. (6).

A third complicating issue is respiratory and cardiac motion, which can cause image blurring. The motion blurring, similar to partial-volume effect (PVE) discussed below, can subsequently lead to overestimation of myocardium tracer uptake in early dynamic frames and underestimation in later frames. On the other hand, the input function derived from blood-pool activity could be underestimated in early frames and overestimated in later frames. In clinical studies, vasodilators may also introduce variations in breathing or irregular breathing amplitudes over time, further complicating the quantification of cardiac images. The CZT SPECT system used in this study is a fully stationary system and therefore can provide consistent angular sampling over all projections, overcoming one of the major limitations of using conventional step-and-shoot SPECT for dynamic imaging. Recent studies have demonstrated the feasibility of dual respiratory and cardiac gating for more conventional static imaging on this stationary SPECT system (14), although additional investigations are needed to develop dual-motion-correction methods for dynamic imaging applications.

The final technical issue that needs to be addressed in absolute quantification of myocardial flow with kinetic modeling of dynamic SPECT images is the PVE. In kinetic modeling, even though a partial-volume factor can be fitted to partially compensate the PVE, it would be interesting to investigate whether image-based partialvolume correction (PVC) as a preprocessing step can further improve the quantification of dynamic SPECT imaging. In cardiac SPECT/CT and PET/CT, contrast-enhanced CT is needed to perform anatomic-based PVC. Typical voxel-based PVC methods amplify image noise, which is a greater degrading factor for dynamic imaging than static imaging, because of the low count level in each dynamic image. Recently developed PVC approaches that can simultaneously correct for PVE and reduce image noise are promising and need further investigation in dynamic SPECT and PET imaging to improve quantitative accuracy (15).

The use of stationary high-sensitivity solid-state CZT SPECT imaging systems for dynamic SPECT imaging holds tremendous promise for the estimation of absolute myocardial flow and flow reserve if all of these technical issues can be addressed. This novel dynamic SPECT imaging approach could provide a noninvasive approach for the estimation of absolute myocardial flow and flow reserve similar to PET imaging, offering an improved diagnostic accuracy and prognostic value of stress perfusion imaging.

So is the assessment of absolute myocardial perfusion with dynamic SPECT imaging ready for prime time? Almost, if we carefully address all of the resolvable technical issues associated with dynamic SPECT imaging. However, the real application of quantitative dynamic SPECT will come with the availability of ${ }^{99 \mathrm{~m}}$ Tc-labeled radiotracers with more favorable kinetics that if used in combination with high-sensitivity solid-state SPECT imaging systems could provide a truly quantitative assessment of myocardial perfusion with low radiation exposure. 


\section{DISCLOSURE}

No potential conflict of interest relevant to this article was reported.

Chi Liu

Yale University School of Medicine New Haven, Connecticut

Albert J. Sinusas Yale University School of Medicine New Haven, Connecticut

\section{REFERENCES}

1. Murthy VL, Di Carli MF. Non-invasive quantification of coronary vascular dysfunction for diagnosis and management of coronary artery disease. $\mathrm{J} \mathrm{Nucl}$ Cardiol. 2012;19:1060-1072, quiz 1075.

2. Tahari AK, Lee A, Rajaram M, et al. Absolute myocardial flow quantification with ${ }^{82} \mathrm{Rb}$ PET/CT: comparison of different software packages and methods. Eur J Nucl Med Mol Imaging. 2014;41:126-135.

3. Gullberg GT, Reutter BW, Sitek A, Maltz JS, Budinger TF. Dynamic single photon emission computed tomography: basic principles and cardiac applications. Phys Med Biol. 2010;55:R111-R191.

4. Gullberg GT, Di Bella EV, Sinusas AJ. Estimation of coronary flow reserve: can SPECT compete with other modalities? J Nucl Cardiol. 2001;8:620-625.
5. Ben-Haim S, Murthy VL, Breault C, et al. Quantification of myocardial perfusion reserve using dynamic SPECT imaging in humans: a feasibility study. J Nucl Med. 2013;54:873-879.

6. Wells RG, Timmins R, Klein R, et al. Dynamic SPECT measurement of absolute myocardial blood flow in a porcine model. J Nucl Med. 2014;55:1685-1691.

7. Sugihara H, Yonekura Y, Kataoka K, Fukai D, Kitamura N, Taniguchi Y. Estimation of coronary flow reserve with the use of dynamic planar and SPECT images of Tc-99m tetrofosmin. $J$ Nucl Cardiol. 2001;8:575-579.

8. Iida H, Eberl S. Quantitative assessment of regional myocardial blood flow with thallium-201 and SPECT. J Nucl Cardiol. 1998;5:313-331.

9. Smith AM, Gullberg GT, Christian PE, Datz FL. Kinetic modeling of teboroxime using dynamic SPECT imaging of a canine model. J Nucl Med. 1994;35:484-495.

10. Reutter BW, Gullberg GT, Huesman RH. Kinetic parameter estimation from attenuated SPECT projection measurements. IEEE Trans Nucl Sci. 1998;45:30073013.

11. Holstensson M, Erlandsson K, Ben-Haim S, Hutton B. Correction for scatter in ${ }^{99 \mathrm{~m} T c}$ imaging for a CZT-based cardiac SPECT scanner [abstract]. J Nucl Med. 2012;53(suppl 1):2239.

12. Fan $\mathrm{P}$, Hutton BF, Ljungberg $\mathrm{M}$, et al. Scatter and crosstalk corrections for ${ }^{99 \mathrm{~m}} \mathrm{Tc} /{ }^{123} \mathrm{I}$ dual-isotope imaging using a CZT SPECT system with pinhole collimators. IEEE Nucl Sci Symp Med Imaging Conf. In press.

13. Chan C, Dey J, Sinusas A, Liu C. Improved image reconstruction for cardiac dedicated multiple pinhole SPECT with truncated projections. J Nucl Med. 2012;53(suppl 1):105.

14. Chan C, Harris M, Le M, et al. End-expiration respiratory gating for a high resolution stationary cardiac SPECT system. Phys Med Biol. In press.

15. Chan C, Liu H, Grobshtein Y, Stacy MR, Sinusas AJ, Liu C. Simultaneous partial volume correction and noise regularization for cardiac SPECT/CT. IEEE Nucl Sci Symp Med Imaging Conf. 2013:1-6. 